\title{
A GENERALIZATION OF NONHARMONIC FOURIER SERIES
}

\author{
HAROLD E. BENZINGER
}

(Communicated by Paul S. Muhly)

\begin{abstract}
It is shown that the theory of nonharmonic Fourier series is a special case of a general method for perturbing bases in Banach spaces, and that even in the classical case, there are many ways of perturbing ordinary Fourier series while still preserving the norm and pointwise convergence properties.
\end{abstract}

\section{INTRODUCTION}

The theory of nonharmonic Fourier series is concerned with placing conditions on a sequence of complex numbers $\delta_{n},-\infty<n<\infty$, such that the sequence of functions $g_{n}(x)=e^{i\left(n+\delta_{n}\right) x}$ has the same expansion properties as the sequence $\phi_{n}(x)=e^{i n x}$, with respect to convergence in the norm of $L^{p}(-\pi, \pi)$, $1 \leq p<\infty$, or with respect to pointwise convergence. In [1] it was shown that if $\left\{\delta_{n}\right\}$ is assumed to be a Fourier series multiplier sequence, then nonharmonic Fourier series arise as the eigenfunctions of unbounded linear operators with useful spectral properties, e.g. the operators generate strongly continuous groups or semigroups, and that many earlier results can be unified and extended. In this paper the process of forming the sequence $\left\{g_{n}\right\}$ from $\left\{\phi_{n}\right\}$ is given in an abstract setting, not dependent on the special nature of the exponential function. The results on norm convergence give intrinsic meaning to the hypotheses needed in the classical case. Restricting consideration to the function spaces $L^{p}(-\pi, \pi)$, we see that the perturbation of ordinary Fourier series can be carried out in many ways other than that given above, while obtaining results on pointwise as well as norm convergence.

References to earlier work can be found in $[1,2]$.

\section{NORM CONVERGENCE}

Let $B$ denote a complex Banach space and let $\left\{\phi_{n}\right\},-\infty<n<\infty$ denote a sequence in $B$. Let

$$
w(z)=\sum_{k=0}^{\infty} w_{k} z^{k}
$$

Received by the editors August 10, 1988.

1980 Mathematics Subject Classification (1985 Revision). Primary 42C15, 42A45. 
be an analytic function with radius of convergence $\rho, 0<\rho \leq \infty$, and let

$$
\tilde{w}(z)=\sum_{k=0}^{\infty}\left|w_{k}\right| z^{k}
$$

be the majorizing function of $w$, also with radius of convergence $\rho$. Let $X: B \rightarrow B$ be a bounded linear operator and let $\left\{\delta_{n}\right\}$ be a sequence of complex numbers. For each $n$, let

$$
w\left(i \delta_{n} X\right)=\sum_{k=0}^{\infty} w_{k}\left(i \delta_{n} X\right)^{k}
$$

which exists as a bounded linear operator provided

$$
\left|\delta_{n}\right|\|X\|<\rho .
$$

2.1. Definition. $\left\{\delta_{n}\right\}$ is a multiplier sequence for $\left\{\phi_{n}\right\}$ if there exists a bounded linear operator $M: B \rightarrow B$ such that

$$
M \phi_{n}=\delta_{n} \phi_{n}
$$

for each $n$. Note that $\left|\delta_{n}\right| \leq\|M\|$ for each $n$.

Assuming (2.4) holds, let

$$
g_{n}=w\left(i \delta_{n} X\right) \phi_{n} .
$$

2.2. Theorem. Assume $\left\{\delta_{n}\right\}$ is a multiplier sequence for $\left\{\phi_{n}\right\}$ and

$$
\|X\|\|M\|<\rho .
$$

Then there exists a bounded linear operator $A: B \rightarrow B$ such that

$$
A \phi_{n}=g_{n} \text {. }
$$

Proof. Let

$$
A=\sum_{k=0}^{\infty} w_{k} i^{k} X^{k} M^{k}
$$

This series converges by (2.7) and $\|A\| \leq \tilde{w}(\|X\|\|M\|)$. One sees directly that (2.8) holds.

2.3. Examples. Let $B=L^{p}(-\pi, \pi)$ for $1 \leq p<\infty$, with dual space $B^{*}=$ $L^{q}(-\pi, \pi)$, where $p q=p+q$. Let $(f, g)=(1 / 2 \pi) \int_{-\pi}^{\pi} f(x) \bar{g}(x) d x$, and let $\phi_{n}(x)=e^{i n x}$ in $B, \psi_{n}(x)=e^{i n x}$ in $B^{*}$. Let $X f(x)=h(x) f(x)$ for some $h$ in $L^{\infty}(-\pi, \pi)$. Then $g_{n}(x)=w\left(i \delta_{n} h(x)\right) \phi_{n}(x)$, provided $\left|\delta_{n}\right|\|h\|_{\infty}<\rho$. The classical case of nonharmonic Fourier series arises from $h(x)=x$ and $w(z)=e^{z}$. Then $\rho=\infty$ and $g_{n}(x)=e^{i\left(n+\delta_{n}\right) x}$. Clearly many other cases can be considered, such as $h(x)=x^{2}$, and then one is concerned with the expansion properties of

$$
g_{n}(x)=e^{i\left(n x+\delta_{n} x^{2}\right)}
$$


If $w(z)=1 /(1-z), h(x)=x$, then $\rho=1$,

$$
g_{n}(x)=\left(1-i \delta_{n} x\right)^{-1} \phi_{n}(x),
$$

and $A$ exists if $\pi\|M\|<1$.

We return to the abstract setting.

2.4. Definition. The sequence $\left\{g_{n}\right\}$ is equivalent to the sequence $\left\{\phi_{n}\right\}$ if $A$ has a bounded inverse.

If the two sequences are equivalent then all topological properties of expansions in one system are transplanted to the other. In particular, if $\left\{\phi_{n}\right\}$ is linearly independent and complete in $B$ and if there exists a sequence $\left\{\psi_{n}\right\}$ in the dual space $B^{*}$ such that $\left(\phi_{n}, \psi_{n}\right)=\delta_{n m}$, then $\left\{g_{n}\right\}$ is complete and linearly independent and defining

$$
h_{n}=A^{*-1} \psi_{n},
$$

we have $\left(g_{n}, h_{m}\right)=\delta_{n m}$. If $\left\{\phi_{n}\right\}$ is a Schauder basis for $B$, then so is $\left\{g_{n}\right\}$. To give explicit conditions for $A$ to have a bounded inverse, we use the geometric series method and the Fredholm method: $A$ has a bounded inverse if $\|A-I\|<$ 1 , or if $A-I$ is compact and $A$ is one-to-one.

2.5. Theorem. A sufficient condition for $A$ to have a bounded inverse is that

$$
\tilde{w}(\|X\|\|M\|)<1+\left|w_{0}\right|-\left|1-w_{0}\right| .
$$

Proof. From the definition of $A$ in (2.11) we see that

$$
\|A-1\| \leq\left|1-w_{0}\right|-\left|w_{0}\right|+\tilde{w}(\|X\|\|M\|) .
$$

Using (2.12), this yields $\|A-I\|<1$.

If $w_{0}=1$ then $(2.12)$ becomes

$$
\tilde{w}(\|X\|\|M\|)<2 .
$$

If $w(z)=e^{z}=w_{M}(z)$, this condition becomes

$$
\|M\|<\ln 2 /\|X\|,
$$

and if $X f(x)=x f(x)$ in $L^{p}(-\pi, \pi)$, then $A$ has a bounded inverse if

$$
\|M\|<\ln 2 / \pi .
$$

See Theorem 2.11 in [1] and the remarks there about earlier results of Duffin and Eachus and of Pollard. For the system given in (2.10), $A$ exists if $\|M\|<1 / \pi^{2}$, and a sufficient condition for the invertibility of $A$ is that $\|M\|<\ln 2 / \pi^{2}$. For the system given in (2.11), $A$ is invertible if $\|M\|<1 / 2 \pi$.

To apply the Fredholm method, we need to have $A-I$ compact. 
2.6. Proposition. If $M$ is compact then $A-I$ is compact.

Proof. All terms in (2.9) for $k>0$ are compact operators, and then $A-I$ is the limit in the operator norm of compact operators.

Since $g_{n}=\phi_{n}$ if $\delta_{n}=0$, it suffices to consider perturbing only finitely many vectors at a time. From Lemma 2.14 of [1] we have

2.7. Proposition. Assume (2.7) and that $\delta_{n}=0$ except for $n$ in a finite set S. Let

$$
D_{S}=\operatorname{det}\left[\left(g_{n}, \psi_{m}\right)\right], \quad n, m \text { in } S .
$$

Then $A$ is invertible if $D_{S} \neq 0$.

Note that $D_{S}$ is an analytic function of $\delta_{j}$ for each $j$ in $S$, and $D_{S} \neq 0$ when all $\delta_{j}=0$. Thus although the zero set of $D_{S}$ can be uncountable, there is no constraint on the size of the deltas, since there are values arbitrarily close to the circle of convergence which are not in the zero set of $D_{S}$. Using the geometric and Fredholm methods, we obtain a condition with no constraint on the sizes of all of the $\delta$ 's.

2.8. Theorem. Let $M$ be a compact multiplier satisfying (2.9). Assume there exists a finite set $S$ of indices such that $D_{S} \neq 0$. Let $M_{S}$ denote the multiplier transform obtained by replacing $\delta_{n}$ by zero for $n$ in $S$. Assume $\tilde{w}\left(\|X\|\left\|M_{S}\right\|\right)<$ 1. Then $\left\{g_{n}\right\}$ and $\left\{\phi_{n}\right\}$ are equivalent.

There exist multiplier transforms of arbitrarily small norm for which $\left\{\delta_{n}\right\}$ does not converge to zero, so the equivalence of two sequences of vectors does not require a constraint on the ultimate size of the multiplier sequence.

In the case that $\left\{\phi_{n}\right\}$ is linearly independent and complete, and there exists a dual sequence $\left\{\psi_{n}\right\}$, there is a condition for the compactness of $M$.

2.9. Proposition. $M$ is compact if

$$
\sum_{-\infty}^{\infty}\left|\delta_{n}\right|\left\|\phi_{n}\right\|\left\|\psi_{n}\right\|<\infty
$$

Proof. This condition ensures that $M$ is the limit in the operator norm of the finite rank operators

$$
M_{N} f=\sum_{-N}^{N} \delta_{n}\left(f, \psi_{n}\right) \phi_{n}
$$

Condition (2.22) can always be satisfied by putting strong conditions on the multiplier sequence. In the case of classical Fourier series, a less stringent condition can be given, using the Hausdorff-Young inequality.

2.10. Proposition. Let $B=L^{p}(-\pi, \pi)$ for $1<p \leq 2$. M is compact if $\left\{\delta_{n}\right\}$ is in $l^{p}$. 


\section{Stability}

A perturbation of $\left\{\phi_{n}\right\}$ is determined by specifying a power series and two bounded linear operators, one of which is a multiplier. Assume $w_{0}, X_{0}, M_{0}$ have been selected so that $A_{0}$ exists and has a bounded inverse. We show that for any triple $w, X, M$ sufficiently close to $w_{0}, X_{0}, M_{0}$, the resulting operator $A$ has a bounded inverse. For $X, M$ closeness is measured using the operator norm. Two power series $w(z), w_{0}(z)$ are close if their respective radii of convergence $\rho, \rho_{0}$ are close and if

$$
\sum_{k=0}^{\infty} \gamma_{X}^{k} \gamma_{M}^{k}\left|w_{k}-w_{k}^{0}\right|
$$

is sufficiently small, where $\gamma_{X}, \gamma_{M}$ are positive constants determined by $w_{0}$, $X_{0}, M_{0}$.

By assumption $A_{0}$ is bounded, so $\left\|X_{0}\right\|\|M\|<\rho_{0}$. Select $\varepsilon>0$ such that

$$
\left(\left\|X_{0}\right\|+\varepsilon\right)\left(\left\|M_{0}\right\|+\varepsilon\right)<\rho_{0}-\varepsilon
$$

and then select $w, X, M$ such that $\rho>\rho_{0}-\varepsilon,\left\|X-X_{0}\right\|<\varepsilon,\left\|M-M_{0}\right\|<\varepsilon$. Let $\gamma_{X}=\left\|X_{0}\right\|+\varepsilon, \gamma_{M}=\left\|M_{0}\right\|+\varepsilon$. Then $\|X\|\|M\|<\rho$, so $A$ exists as a bounded linear operator. Let $\left.a_{k}=\max \left(\mid w_{k}\right),\left|w_{k}^{0}\right|\right)$.

We make use of the identity for linear operators

$$
P Q-R S=\frac{1}{2}(P+R)(Q-S)+\frac{1}{2}(P-R)(Q+S) .
$$

3.1. Lemma. Let $T, T_{0}$ be bounded linear operators and let $\gamma=$ $\max \left(\|T\|,\left\|T_{0}\right\|\right)$. Then

$$
\left\|T^{k}-T_{0}^{k}\right\| \leq k \gamma^{k-1}\left\|T-T_{0}\right\| .
$$

Proof. Let $P=T^{k-1}, Q=T, R=T_{0}^{k-1}, S=T_{0}$. Then from (3.1),

$$
\left\|T^{k}-T_{0}^{k}\right\| \leq \gamma^{k-1}\left\|T-T_{0}\right\|+\gamma\left\|T^{k-1}-T_{0}^{k-1}\right\| .
$$

The result follows by induction.

\subsection{Lemma.}

$$
\begin{aligned}
\left\|w_{k} X^{k} M^{k}-w_{k}^{0} X_{0}^{k} M_{0}^{k}\right\| \leq & a_{k} \gamma_{X}^{k}\left\|M^{k}-M_{0}^{k}\right\| \\
& +a_{k} \gamma_{M}^{k}\left\|X^{k}-X_{0}^{k}\right\|+\left(\gamma_{X} \gamma_{M}\right)^{k}\left|w_{k}-w_{k}^{0}\right| .
\end{aligned}
$$

Proof. Apply (3.1) twice, first with $P=w_{k} X^{k}, Q=M^{k}$, etc., and then with $P=w_{k}, Q=X^{k}$, etc.

\subsection{Proposition.}

$$
\begin{aligned}
\left\|A-A_{0}\right\| \leq & \sum_{k=0}^{\infty}\left(\gamma_{X} \gamma_{M}\right)^{k}\left|w_{k}-w_{k}^{0}\right| \\
& +\left[\gamma_{M}\left\|X-X_{0}\right\|+\gamma_{X}\left\|M-M_{0}\right\|\right] \sum_{k=0}^{\infty} k a_{k}\left(\gamma_{X} \gamma_{M}\right)^{k} .
\end{aligned}
$$


Proof. This follows from (3.2) and (3.3) applied to

$$
A-A_{0}=\sum_{k=0}^{\infty} i^{k}\left[w_{k} X^{k} M^{k}-w_{k}^{0} X_{0}^{k} M_{0}^{k}\right]
$$

3.4. Theorem. If $A_{0}$ has a bounded inverse, then there exists $\varepsilon>0$ such that if $\left\|X-X_{0}\right\|<\varepsilon,\left\|M-M_{0}\right\|<\varepsilon, \rho>\rho_{0}-\varepsilon$ and $\sum_{k=0}^{\infty}\left(\gamma_{X} \gamma_{M}\right)^{k}\left|w_{k}-w_{k}^{0}\right|<\varepsilon$, then $A$ has a bounded inverse.

Proof. This follows from (3.7) and the fact that the invertible operators form an open subset of the set of bounded operators.

3.5 Remark. Let $B$ be a Hilbert space and let $w=w_{0}, X=X_{0}$. If $\left\{\phi_{n} /\left\|\phi_{n}\right\|\right\}$ is a Riesz basis for $B$, then $\left\{\delta_{n}\right\},\left\{\delta_{n}^{0}\right\}$ are multiplier sequences if and only if they are bounded, and $\left\|M-M_{0}\right\|=\sup \left|\delta_{n}-\delta_{n}^{0}\right|$. Thus if $M_{0}$ generates an invertible $A_{0}$ then there exists $\Delta>0$ such that for all $\delta_{n}$ with $\left|\delta_{n}-\delta_{n}^{0}\right|<\Delta$, $M$ also generates an invertible $A$. This case was considered by Young in [3] for $B=L^{2}(-\pi, \pi), w(z)=e^{z}$, and $X$ the operation of multiplication by the independent variable.

\section{Pointwise convergence}

We restrict consideration to the case that $\left\{\phi_{n}\right\}$ is the trigonometric system $\left\{e^{i n x}\right\}$ in $B=L^{p}(-\pi, \pi), 1 \leq p<\infty$, and $X f(x)=h(x) f(x)$ for $h$ in $L^{\infty}(-\pi, \pi)$. Let the partial sum operators be

$$
S_{N} f=\sum_{-N}^{N}\left(f, \psi_{n}\right) \phi_{n}, \quad \widetilde{S}_{N} f=\sum_{-N}^{N}\left(f, h_{n}\right) g_{n} .
$$

Assuming $\left\{g_{n}\right\},\left\{\phi_{n}\right\}$ are equivalent, we have

$$
\left(\tilde{S}_{N}-S_{N}\right) f=\left[\sum_{k=1}^{\infty}\left(X^{k} S_{N}-S_{N} X^{k}\right) w_{k}(i M)^{k}\right] A^{-1} f .
$$

See the proof of Theorem 3.1 in [1], where $w_{k}=1 / k$ ! .

4.1 Theorem. Let $p$ be fixed, $1 \leq p<\infty$. Assume $\left\{g_{n}\right\},\left\{\phi_{n}\right\}$ are equivalent and $h$ is of class $C^{2}$. Then for each $f$ in $L^{p}(-\pi, \pi)$,

$$
\lim _{N \rightarrow \infty}\left[\tilde{S}_{N}(x ; f)-S_{N}(x ; f)\right]=0
$$

uniformly on each interval $[-\pi+d, \pi+d], d>0$.

4.2 Remark. This is a generalization of Theorem 3.1 of [1].

4.3 Proof of Theorem 4.1. Let $D_{N}$ denote the Dirichlet kernal

$$
D_{N}(x-t)=\frac{\sin \left(N+\frac{1}{2}\right)(x-t)}{2 \sin ((x-t) / 2)} \text {. }
$$


Then for any $g$ in $B$,

$$
\left(X^{k} S_{N}-S_{N} X^{k}\right) g(x)=\int_{-\pi}^{\pi} D_{N}(x-t)\left[h^{k}(x)-h^{k}(t)\right] g(t) d t .
$$

We use the identity

$$
h^{k}(x)-h^{k}(t)=(h(x)-h(t)) \sum_{l=0}^{k-1} h^{k-l-1}(x) h^{1}(t) .
$$

For $|x| \leq \pi-d$, there exists a constant $K=K_{d}$ such that

$$
\left|\frac{h(x)-h(t)}{2 \sin ((x-t) / 2)}\right| \leq K \text {. }
$$

Then $\left|\left(X^{k} S_{N}-S_{N} X^{k}\right) g(x)\right| \leq K k\|X\|^{k-1}\|g\|$ and for any integer $J$,

$$
\left|\sum_{J}^{\infty}\left(X^{k} S_{N}-S_{N} X^{k}\right) w_{k}(i M)^{k} A^{-1} f\right| \leq K\|M\|\left(\sum_{J}^{\infty} k\left|w_{k}\right|(\|X\|\|M\|)^{k-1}\right)\|f\| \text {. }
$$

Thus given $\varepsilon>0, J$ can be selected so large that the above expression does not exceed $\varepsilon / 2$. For the finitely many remaining terms, since $h$ is in $C^{2}$, $[h(x)-h(t)] / \sin ((x-t) / 2)$ has a derivative with respect to $t$ which is bounded uniformly with respect to $x$ and $t$ for $|x| \leq \pi-d$ and $|t| \leq \pi$. Thus the Riemann-Lebesgue lemma applies uniformly in $x,|x| \leq \pi-d$, and by selecting $N$ sufficiently large, we have

$$
\left|\widetilde{S}_{N}(x ; f)-S_{N}(x ; f)\right|<\varepsilon .
$$

Ordinary Fourier series arise as the eigenfunction expansions of the differential operator $u \rightarrow-i u^{\prime}$ with periodic boundary conditions on $[-\pi, \pi]$. The considerations in $[1, \S 4]$ apply without change to the more general case considered here (even if the pointwise results do not hold), so there is a closed, densely defined operator $\Lambda$ with $\left\{g_{n}\right\}$ as eigenvectors, such that $i \Lambda$ is the infinitesimal generator of a uniformly bounded, strongly continuous semigroup, and $-\Lambda^{2}$ is the infinitesimal generator of a strongly continuous semigroup.

\section{REFERENCES}

1. H. E. Benzinger, Nonharmonic Fourier series and spectral theory, Trans. Amer. Math. Soc. 299 (1987), 245-259.

2. R. M. Young, An introduction to nonharmonic Fourier series, Academic Press, New York, 1980.

3. R. M. Young, On the stability of exponential bases in $L^{2}(-\pi, \pi)$, Proc. Amer. Math. Soc. 100 (1987), 117-122.

Department of Mathematics, University of Illinois, 1409 W. Green Street, Urbana, ILLINOIS 61801 